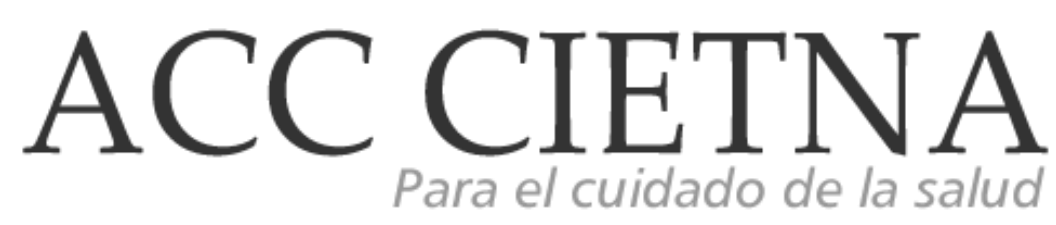

https:// doi.org/10.35383/cietna.v8i1 1.437

ENSAYO

\title{
Cultura de vida y repercusión en la docencia de enfermería
}

\author{
Elizalde Ordóñez Humberto', Cervera Vallejos Mirtha Flor², Aya Roa Kevin Julián³, Peláez Abad María \\ Astrid4, Guamán Castillo Amada Balbina $5^{5}$
}

\begin{tabular}{ll}
\hline INFORMACIÓN DEL ARTíCULO & RESUMEN \\
\hline Historia del artículo: & Todo ser humano, donde se encuentre, siempre está inmerso y en \\
Recibido el 11 de septiembre de 2020 & relación dinámica con la cultura que lo rodea, sean personas diversas, \\
Aceptado el 11 de febrero de 2021 & o universitarios, entonces la misión de la docencia es formar personas \\
\hline Palabras clave: & conscientes de su mundo y de lo que son capaces de hacer a favor de \\
Cultura & este. Este ensayo permitió dilucidar sobre la cultura de vida vinculada a \\
Vida & la docencia de enfermería, concluyendo que la desestructuración del \\
Docencia & proceso pedagógico de enfermería sin cultura de vida, resta el espíritu \\
Enfermería & de superación de estudiantes con expectativas de progreso, porque se \\
& considera a veces a la ignorancia como la única e inequívoca expresión \\
& de dominio.
\end{tabular}

Culture of life and impact on nursing teaching

\section{ABSTRACT}

Keywords:

Culture

Life

Teaching

Nursing
Every human being, wherever they are, is always immersed and in a dynamic relationship with the culture that surrounds them, whether they are diverse people or university students, so the mission of teaching is to train people who are aware of their world and what they are capable of doing for it. This essay allowed to elucidate on the culture of life linked to the teaching of nursing, concluding that the destructuring of the pedagogical process of nursing without culture of life, subtracts the spirit of improvement of students with expectations

\footnotetext{
1 Magíster en Enfermería Clínico-Quirúrgica. Área Biológica-Biomédica. Docente de la Universidad Técnica Particular de Loja, Ecuador. Email: helizalde@utpl.edu.ec. ORCID: https://orcid.org/0000-0002-3157-5603

2Doctora en Ciencias de Enfermería. Maestra en Formación Directiva en Gobierno de Personas. Maestra en ciencias con mención en Salud Pública. Maestra en Bioética. Enfermera Especialista en Nefrología, Chiclayo, Perú. Email: mcervera@usat.edu.pe. ORCID: https://orcid.org/0000-0002-4972-1787

${ }^{3}$ Candidato a Maestro en Ciencias de Enfermería. Universidad Guanajuato, México. Docente catedrático. Facultad de estudios superiores Zaragoza. Universidad Nacional Autónoma de México. Email: kjayaroa@ugto.mx. ORCID: https://orcid.org/0000-0002-8430-078

4 Magíster en Enfermería Clínico-Quirúrgica. Área Biológica-Biomédica. Docente de la Universidad Técnica Particular de Loja, Ecuador. Email: mapelaez55@utpl.edu.ec. ORCID: https://orcid.org/0000-0002-9651-3673

${ }_{5}^{5}$ Magíster en Gerencia para el desarrollo local. Área Biológica-Biomédica. Docente de la Universidad Técnica Particular de Loja, Ecuador. email abguaman1@utpl.edu.ec. ORCID: https://orcid.org/0000-0003-2149-636X
} 
of progress, because ignorance is sometimes considered as the only and unequivocal expression of mastery.

\section{Introducción}

Existe cierta dificultad al detallar conceptualizaciones, que enmarcan la realidad del comportamiento, la relación entre los seres humanos y la apertura de comunicación en la diversidad cultural, porque estas acciones realizadas por las personas son consecuencia de la libertad, como lo explica Herranz ${ }^{1}$ una cultura de vida, necesita generosidad, servicio, abandonar el egoísmo y decidirse por la preocupación constante por los demás.

Esto es saber elegir, y se sabe elegir cuando se conoce a profundidad lo que anhelados $o$ queremos, de lo contrario decimos y actuamos de manera incoherente y sin argumentación, especialmente cuando se trata de abordar temas profundos como cultura de la vida, docencia o enfermería, en una sociedad en crisis y con ideales egocéntricos, que desvía el centro de las acciones hacia los más vulnerables empezando por el ser engendrado, luego a las personas de cualquier raza, situación social, política, edad, educación o religión en su multidimensionalidad y multiculturalidad con la finalidad de asegurar $y$ proteger su dignidad ${ }^{2,3}$.

Aspectos que deben ser entendidos y enseñados específicamente en la docencia de enfermería donde existen modelos que no emanan una verdadera comprensión y significado de lo que es la persona desde que es engendrada hasta su término natural, de allí que el objetivo acogido en este ensayo fue dilucidar cómo la cultura de la vida está anclada en la docencia de enfermería. Y optar por una cultura de vida es inevitable, ya que en su esencia comprende un derecho y un deber que tiene que ser querido, protegido y difundido especialmente por los docentes de enfermería.

Razonamos que la docencia de enfermería considera la característica humana que identifica su labor, asociando cultura de vida, puesto que la cuida y protege en todo su ciclo vital en su multidimensionalidad, es decir tocando el aspecto cultural de cada ser cuidado, sin embargo, los enlaces figurativos que no se evidencian en la formación, evoca sensaciones extrañas, que difícilmente se pueden explicar con palabras, ante la contemplación de tantas desigualdades.

De allí, no solo optar por una educación innovadora sino también la renovación y praxis de una legislación que regularice la vida humana con términos protectores y respetuosos con la misma, pues todo lo relacionada a la vida afecta a la dignidad y a la propia esencia e identidad ${ }^{4}$.

Entonces cabe aludir como la formación del futuro profesional de enfermería, cursa grandes innovaciones porque en la variedad de investigaciones existentes se ha llegado a contemplar a la persona en cualquier situación de salud como un ser biopsicosocial, necesitado de la ciencia como entender que posee diferentes tipos de creencias, las cuales determinan su actuar y la aceptación o no de determinados tratamientos para conservar la vida como lo argumenta Valencia 5 .

\section{Desarrollo}

\section{Cultura de vida}

Argumentado, la familiaridad del contexto social y laboral en el ejercicio docente de los enfermeros denota la estrecha correlación vivencial de un entorno vulnerable en aspectos movidos a esferas tangibles de superioridad o bondad, creando sinergia, frente a sembrar dudas en cómo será la dinámica de cada encuentro. La enseñanza en su quehacer moral irrumpe como acción humana con libertad inteligencia y voluntad llevada a cabo con otros seres humanos en donde están siempre presentes cuestiones referidas a lo que les sea justo y peculiar6. 
La peculiaridad razonada del proceso de formación y la práctica docente, centrada en la relación y el establecimiento de acción-reacción garantizan cuidar las derivaciones esenciales inmiscuidas, que figuren en el proceso de cultura de vida en la docencia de enfermería.

La cultura de vida enfatiza Melguizo ${ }^{7}$ enrola evidencias de defender la vida en cualquier contexto y en la variedad social, así como en cada una de sus expresiones. Concibiendo que cultura es la forma como se expresa una sociedad en sus valores, convicciones y capacidades. Una cultura de la vida visibiliza lo que las personas expresan en bien de todo aquello que es vida.

Porque, el ser humano, en el contexto donde se ubique, siempre está inmerso y en relación dinámica con la cultura que lo rodea. Con lo cual, es imprescindible desarrollar la practica desde la esencia de lo individual respetando los cánones de comportamiento de manera propia en los límites de arraigo, idiosincrasia y costumbres, con la posibilidad de permitir la libertad de cada persona hacia la inclusión en el grupo fortaleciendo el desempeño en las labores de atención y capacitación. Pero también avalar la intervención de los estudiantes en los procesos de toma de decisiones educativas y aumentar la colaboración de los estudiantes en las actividades sociales 8,9 .

La práctica docente y el cuidado basado en respeto a la cultura se remonta a los inicios de la profesión, ya que el cuidado es el objeto de estudio de la disciplina, práctica oportuna para la atención de la persona, familia y comunidad en su experiencia de salud, con los aportes teóricos de los diferentes paradigmas. De esta manera con la globalización y las nuevas políticas socioeconómicas, los profesionales de enfermería son capaces de desenvolverse ante los diversos cambios sociales, políticos y económicos, posesionándose con retos ante el cuidado con calidad e integralidad que se les brinda a las personas y a su entorno. Basado en lo anterior, nunca debe ponerse en tela de duda, en relación con el esfuerzo realizado por el educador, y menos minimizar el esfuerzo pedagógico en los tiempos actuales si cabe mencionar, que en varios escenarios podría caerse en la arrogancia, cuando tales acciones no brindan garantía de aprendizaje para el educando, expresado por Álvarez ${ }^{10}$.

La docencia como la práctica en la educación superior, parte de entender la variedad de acepciones semánticas. El profesional de enfermería es el eje del cambio en las diferentes áreas de aplicación y desempeño, como son la asistencial, la educativa, la administrativa y la de investigación. De esta manera, se revela la diversificación que ha tenido la profesión, producto de años de evolución y de las tendencias que han forjado las bases para el presente, dando continuidad al futuro. Entonces, permite argumentar, que la misión de la docencia es la de formar personas conscientes de su mundo y de lo que son capaces de hacer a favor de ese mundo. La verdadera docencia es aquella que propicia que el alumno se forje la necesidad de aprender por su cuenta y que encuentre en el profesor un guía, un acompañante de travesía para llegar al conocimiento y en el grupo un espacio de encuentro, de intercambio, discusión y confrontación de ideas ${ }^{11}$.

Por otro lado, para reflexionar sobre la práctica docente dentro del conjunto de prácticas, es evidente la referencia al ejercicio normativo, precedido por el bien en fortalecer las habilidades y las destrezas, conjugando, observación, conocimiento previo, que sin duda dan sentido y significación a la práctica. Ampliando criterios, es importante hacer constar, el compromiso humano, inmiscuir respeto, bondad, caridad, considerando la diversidad y la minoría, para evocar lo intrínseco del ser humano, cual es la valía de su ser, aún desconocido por muchos, cuando se relaciona en grupos con los mismos intereses. En esta situación de aprendizaje alude Ospina ${ }^{12}$, se estructura al ser humano culturalmente con la finalidad de equilibrar las diferencias que marca la procedencia, las costumbres, las creencias y adaptarlas en las fases académicas enfatizando la elevación de la 
dignidad humana desde que el ser humano es engendrado hasta su término natural, a esferas que trascienda la esencia del sano compartir entre personas en un mismo grupo con muchas diferencias.

Por tanto, el docente durante el curso de la práctica educativa, pedagógica y didáctica, impele a reflexionar, como este proceso de creación dinámica, y que fue abordado en clases, ayuda a descubrir las huellas de la construcción identitaria-cultural, cuando se proceda a analizar el conocimiento de las dimensiones, expresiones y características, específicas de la docencia en enfermería, que de cierta manera ayuda a entender el sentido y significación de la propia identidad cultural, considerando el respeto a los formandos también como personas portadoras de alta dignidad $^{12}$.

Por esta razón la enseñanza es un quehacer moral, porque es la producción de acciones humanas para otras personas: los estudiantes, con el imperativo de desarrollar un cuidado más humanizado hacia los más vulnerables, defender la vida protegerla, abogar por quienes no pueden hablar por sí mismo y mucho menos defenderse. Realidad que compromete a docentes $y$ estudiantes reconociéndose ambos como personas y con quienes tenemos la misión de cuidar hoy y siempre. Sin embargo, los segundos requieren ser asistidos, ayudado para existir y para lo que están llamados a ser. Para ello, hay que sacar lo mejor del estudiante. Apoyarle en su creatividad y autonomía, ilusionarlo y aprenda a ser fiel a sus ideales amando la propia vida y la de los demás, con apertura de sí mismo hacia lo noble y bueno, no cabe duda con esfuerzo y lucha6,13.

\section{Docencia de enfermería}

Fundamentando las cualidades y realidades de la apreciación de la cultura de la vida en la docencia de enfermería, consideramos que la esencia es la práctica humana, redimida desde la concepción cualitativa, de la acción gozosa de infundir y esparcir el don necesario para que, las virtudes a desarrollar académicamente sean altamente gratificantes. Una educación para el desarrollo humano considera el tipo de hombre que debe formar y, por ende, el tipo de sociedad resultante. Por lo tanto, ha de ser potencializadora de la realización de las necesidades humanas y de las esferas del desarrollo humano, y para ello se requiere de procesos de aprendizaje significativos, es decir, de ambientes de aprendizaje que favorezcan la relación armónica del saber, el hacer y el ser de las personas participantes ${ }^{12}$.

Las ventajas analizadas en contexto cultural de quienes forman nuevas generaciones de personas permiten entender que el respeto y la ayuda, es la base de saber escuchar, lo que atribuye pensar intensamente, que el ego inflamado de orgullo solo sirve para violentar la individualidad evitando la perpetuidad de la bondad y respeto. Sobre todo, un respeto profundo por la cultura de las personas y comunidades, actitud de escucha, capacidad de trabajo en equipo y de ejercer un sano liderazgo y ética profesional ${ }^{14}$. Reflexionando mucho sobre lo imposible de aplicar la dualidad equivocada de, "hoy bien-mañana no", aporta Escobar'15 genera inquietud, dejando cierta intranquilidad, por cuanto el contexto del entorno se ve afectado en actos que pueden ser conscientes e inconscientes, puesto que para regular la conducta humana en los aspectos relacionados con los actos que afectan la vida de las personas y el cuidado de la salud surge la bioética, la cual provee los principios para distinguir entre lo que debe hacerse o no hacerse, proteger y defender la vida, despliegue de principios éticos para que crezca y se desarrolle.

La esperanza, condiciona a mantener la pasividad y no protestar porque existe poco entendimiento, ya que el tiempo reduce y absorbe, entonces nace el interés por profundizar y reflexionar en amplitud sobre cuán, importante es reconocer y hacer de la cultura de la vida, una aproximación de identidad, para comprender y estimar las particularidades culturales. 
Lo cierto es que es un gran desafío, para entender que este proceso del hacer docencia en enfermería, señala Urra ${ }^{16}$ conlleva a un aprendizaje diario de cada estudiante porque enriquece mucho en la parte personal, descubriendo que el vínculo docencia y cultura de la vida, se fundamenta en entender mediante recapitulaciones de desarrollo académico cultural, para coadyuvar a aclarar conocimientos, procedimientos y funciones.

La ciencia de enfermería debería salir fortalecida en la forma de cómo pensar en enfermería, y vendrá una nueva generación denominada productores culturales de enfermería. Lo cierto es que para actuar en base a lo anterior podría ser necesario revisar concepciones, conceptualizaciones y acepciones sobre culturavida-docencia y que estructuralmente favorecerá más que la práctica, el sistema de vida. De allí que el docente con firme convicción en una cultura de vida es un guía de enseñanza para el universitario sobre compromiso humano, donde emerja el respeto, bondad, caridad, para considerar la diversidad y la minoría, cuando se relaciona en grupos con los mismos intereses, de esta manera a los estudiantes se les guía para ilusionarse con la búsqueda de la verdad profunda aún con límites, de lo contrario estos estarán sumergidos con pensares y acciones plagadas de sentimentalismo y emociones, lo cual resulta dañino en el ámbito universitario donde se cultiva el saber superior y acabaran por descaminar no sólo a los docentes, sino también a los futuros enfermeros.

\section{Repercusiones futuras}

La naturaleza humana, nos da sentido de discernimiento, es como algo intrínseco, pero en las fases de desarrollo y crecimiento, se quebranta, por factores asociados ya analizados anteriormente, fomentando desequilibrio e incomprensión, asociada a la objetividad cultural, violentando la vida y provocando malestar en la práctica docente en enfermería.

Muchas veces pueden ser situaciones ajenas o no conscientes acota Rojas ${ }^{17}$, que impiden resaltar las cualidades de cada individuo, porque el ego de una sola persona se inflama de orgullo y vanidad, incluso de irrespeto, frente a otras personas, creando el día a día, con inestabilidad poniendo al borde del precipicio la virtud humana que es la característica principal en la sociedad culturalmente, expresada.

En este sentido, los saberes disciplinares, con las habilidades adquiridas de la propia vivencia en cuanto a ser; ocasiona la desestructuración del proceso pedagógico de enfermería, permanece sometido a criocongelación, mirando desde ópticas diversas, el enlentecimiento del caminar, entonces se minimiza el espíritu de guerreros de un grupo con expectativas de superación, porque se considera a veces a la ignorancia como la única e inequívoca expresión de dominio, creando ilusión y letargo, en las personas con que se unen en un solo grupo, proviniendo de otros grupos asentados en culturas diversas.

Espontáneamente y en contraposición a la época actual, razona Prestes 18 que acciones $y$ pensamientos de análisis de cultura en concordancia con la docencia en enfermería, reafirma el escaso planteamiento y objetividad que los estudiantes argumentan, por la superficialidad de la vivencia, ellos mismo incluyen o excluyen situaciones según la conveniencia, porque también existe la adaptación fácil en acoger costumbres de otras culturas ocultando las propias con la finalidad de mantener el nivel de aceptación en el grupo ocasionando que los procesos de escolarización y aprendizaje de personas adultas en la educación superior y enmarcado en el paradigma de la educación a lo largo de la vida, ha ocasionado reflexionar sobre esta cuestión a partir de enfoques empíricos y explicativos sobre el acceso y la permanencia de estos grupos en el espacio universitario.

De allí que los docentes con competencias en cultura de la vida visionan a la persona y con ello a sus estudiantes en su globalidad y como tal los tratan, con impacto en el hacer de los futuros enfermeros, pues no les resultará aún en crisis 
ofrecer cuidados integrales, adueñándose de las personas enfermas sin distinción alguna, con compasión, respetando las creencias y costumbres de quienes cuidan y también de su familia.

Al respecto, Reluz y Cervera ${ }^{19}$, argumentan que un educador humanista tiene que volver la mirada a las características esenciales del ser humano, en cuanto a persona ubicándose en el propio contexto, sin imitar prototipos que sólo apuntan a la condición de lo humano, brindando libertades contradictorias que atentan contra lo propiamente humano, despersonalizando la humanidad, y queriendo que vaya en extensiva. Como docentes unidos al quehacer universitario en la tarea de formar y cuidar, es necesario construir el conocimiento atendiendo a lo que dignifica $y$ posicionarlo en cada escenario concreto: las instituciones educativas, las clínicas y hospitales. El reto es integrar el conocimiento que, generado en otros tiempos y espacios, apuntaron siempre a un humanismo integrado que dignifica y suscitar nuevas construcciones, contextualizadas en tiempo y espacio actual.

\section{Conclusiones}

La cultura de vida forja situaciones de aprendizaje, que persiguen equilibrar las diferencias que marca la procedencia, las costumbres y, las creencias de las personas, adaptándolas en las fases académicas con énfasis en la elevación de la dignidad humana desde que el ser humano es engendrado hasta su término natural, a esferas que trascienda la esencia del sano compartir entre personas en un mismo grupo con muchas diferencias.

La docencia impartida con cultura de vida busca el desarrollo humano considerando el tipo de persona que debe formarse, con uso de procesos de aprendizaje significativos, es decir, de ambientes de aprendizaje que favorezcan la relación armónica del saber, el hacer y el ser de las personas participantes en su diversidad, sin embargo, aún prevalece las estructuras pedagógicas estandarizadas.
La desestructuración del proceso pedagógico de enfermería resta el espíritu de superación de estudiantes con expectativas de progreso, porque se considera a veces a la ignorancia como la única e inequívoca expresión de dominio, creando ilusión y letargo, en las personas con que se unen en un solo grupo, proviniendo de otros grupos asentados en culturas diversas donde la realidad no es real, y que condicionan la esfera académica - pedagógica.

\section{Bibliografía}

1. Herranz G. La cultura de vida un empeño afirmativo [Internet]. 2001 [Citado 12 Abr 2021]. Disponible en: https://www.almudi.org/noticiasantiguas/2259-la-cultura-de-la-vida-unempeno-afirmativo-gonzalo-herranz-14-32001

2. Guevara B, Evies A, Rengifo J, Salas B, Manrique $D$, Palacio C. El cuidado de enfermería: una visión integradora en tiempos de crisis. Enferm. Glob. [Internet]. 2014 Ene [Citado 12 Abr 2021]; 13(33): 318-327. Disponible en: http://scielo.isciii.es/scielo.php?script=sci_art text\&pid $=$ S1695

$61412014000100016 \& \operatorname{lng}=$ es.

3. Cervera M. Acoger y defender la vida, misión de la Enfermería [Internet]. 2016 [Citado 14 Abr 2021]. Disponible en: http://www.usat.edu.pe/articulos/salud/acog er-y-defender-la-vida-mision-de-laenfermeria/

4. Mateos y de Cabo O. El derecho fundamental a la vida. Cuadernos de Bioética [Internet]. 2021 [Citado 14 Abr 2021]; 32(104): 91-99. Disponible en: http://aebioetica.org/revistas/2021/32/104/ 91.pdf

5. Valencia M, Vega J, Vega T. Universalidad y diversidad cultural, una mirada desde el cuidado de enfermería [Tesis de pregrado en internet]. Cali: Universidad Santiago de Cali; 2020 [Citado 14 Abr 2021]. 61 p. Disponible en: https://repository.usc.edu.co/bitstream/hand 
le/20.500.12421/3702/UNIVERSALIDAD\%20DI

VERSIDAD\%20CULTURAL.pdf? sequence $=4 \&$ is $A$ $\underline{\text { lowed }=\mathrm{y}}$

6. Mansilla J, Ricouz A. Vivencia del rol docente clínico de enfermeras de hospitales del Sur de Chile. Cienc. enferm. [Internet]. 2016 [Citado 29 May 2020]; 22(1): 101-111. Disponible en: https://scielo.conicyt.cl/scielo.php?script=sci _arttext\&pid =S071795532016000100009\&lng=es. http://dx.doi.org/10.4067/S071795532016000100009

7. Melguizo E, Alzate M. Creencias y prácticas en el cuidado de la salud. Avances en Enfermería [Internet]. 2008 [Citado 29 May 2020]; 26(1): 112-123. Disponible en: https://revistas.unal.edu.co/index.php/avenfe $\mathrm{rm} /$ article/view/12891/13649

8. Mondragón-Hernández I, Sosa-Rosas M. Experiencias de los alumnos en el Aprendizaje del Proceso de atención de enfermería. Enfermería universitaria [Internet]. 2019 [Citado 29 May 2020]; 16(3): 249-258. Disponible en: https://dx.doi.org/10.22201/eneo.23958421 e.2019.3.709

9. Kantek F, Baykal U, Altuntas S. Culture of nursing school: students' perceptions. Procedia [Internet]. 2015 [Citado 29 May 2020]; 174 (2015): 1207 - 13 Disponible en: https://doi.org/10.1016/j.sbspro.2015.01.73 $\underline{8}$

10. Álvarez D. Enfermería en América Latina: una mirada al horizonte. Avances en Enfermería [Internet]. 2015 [Citado 29 May 2020]; 33(2): 295-305. Disponible en: https://doi.org/10.15446/av.enferm.v33n2.3 7032

11. Morán P. La docencia como recreación y construcción del conocimiento Sentido pedagógico de la investigación en el aula. Perfiles educativos [Internet] 2004 [Citado 15 Abr 2021]; 26(105-106): 41-72. Disponible en: http://www.scielo.org.mx/scielo.php?script =s ci_arttext\&pid $=$ S01 85 26982004000100003\&lng $=$ es

12. Ospina B. La educación como escenario para el desarrollo humano. Investigación y Educación en Enfermería [Internet]. 2008 [Citado 28 May 2020]; 26(2, Suppl. 1): 12-15. Disponible en: http://www.scielo.org.co/scielo.php?script $=$ sc i_arttext\&pid $=$ S0120 53072008000300001 \&lng=en\&tlng=es.

13. Cervera M. Inclusión de la ética en la formación universitaria del estudiante de enfermería [Tesis de doctorado en internet]. Perú: Universidad Nacional de Trujillo; 2010 [Citado 28 May 2020]. 184p. Disponible en: https://dspace.unitru.edu.pe/bitstream/handl e/UNITRU/5433/Tesis\%20Doctorado\%20-\%20 Mirtha\%20Cervera\%20Vallejos.pdf?sequence $=$ 1 \&isAllowed $=y$

14. Lagoueyte M. El cuidado de enfermería a los grupos humanos. Rev. Univ. Ind. Santander. Salud [Internet]. 2015 [Citado 29 May 2020]; 47(2): 209-213. Disponible en: http://www.scielo.org.co/scielo.php?script $=$ sc i_arttext\&pid $=$ S0121 $08072015000200013 \&$ lng $=$ en.

15. Escobar-Castellanos B, Cid-Henriquez P. El cuidado de enfermería y la ética derivados del avance tecnológico en salud. Acta bioethica [Internet]. 2018 [Citado 10 Feb 2021]; 24(1): 39-46. Disponible en: https://dx.doi.org/10.4067/S1726569X2018000100039

16. Urra E. Avances de la ciencia de enfermería y su relación con la disciplina. Ciencia y enfermería [Internet]. 2009 [Citado 10 Feb 2021]; 15(2), 9$18 . \quad$ Disponible en: https://dx.doi.org/10.4067/s071795532009000200002

17. Rojas J, Rivera L, Morera M. Aspectos pedagógicos en la formación de Enfermería: Revisión integrativa. Investigación y Educación en Enfermería [Internet]. 2018 [Citado 29 May 2020]; 36(3): e03. Disponible en: https://dx.doi.org/10.17533/udea.iee.v36n3 e03 
18. Prestes E, Diniz A. Educación y aprendizaje a lo largo de la vida: los adultos y la enseñanza superior. Sinéctica [Internet]. 2015 [Citado 29 May 2020]; (45): 1-20. Disponible en: http://www.scielo.org.mx/scielo.php?script $=\mathbf{s}$ ci_arttext\&pid=S1665109X2015000200006\&lng=es\&tIng=es.

19. Reluz F, Cervera M. Precisiones del concepto persona para la formación en las ciencias educativas y del cuidado. Revista Universidad y Sociedad [Internet]. 2021 [Citado 29 May 2020]; 13(2): 134-140. Disponible en: https://rus.ucf.edu.cu/index.php/rus/article/ view/1950 